\title{
New approach to finite frequency Filter Design for two-dimensional T-S fuzzy systems
}

\author{
Ben Meziane Khaddouj ${ }^{1, *}$, Abderrahim El-Amrani ${ }^{2, * *}$, and Ismail BOUMHIDI $^{2, * * *}$ \\ ${ }^{1}$ Department of Engineering, Higher Institute of Engineering and Business (ISGA), Fez, Morroco. \\ ${ }^{2}$ LISAC Laboratory, FSDM, Sidi Mohamed Ben Abdellah University, Fes, Morocco.
}

\begin{abstract}
This paper considers the problem of filter design for two-dimensional (2D) discrete-time non-linear systems in Takagi-Sugeno (T-S) fuzzy mode. The problem to be solved in the paper is to find a $H_{\infty}$ filter model such that the filtering error system is asymptotically stable. A numerical example is employed to illustrate the validity of the proposed methods.
\end{abstract}

\section{Introduction}

In the last few decades, many researchers have investigated two-dimensional (2-D) systems including continuous, discrete and continuous-discrete settings as these systems have great applications in engineering fields such as process control, multi-dimensional digital filtering, image processing, see for instance [1-6].

Among the most of the existed literature on filtering problems, the disturbances are considered in the entire frequency (EF) domain, which will bring over design in the filtering design. While many practical engineering problems are more suitable to be considered in finite frequency (FF) ranges.. So, the standard design approaches for the whole frequency domain may bring conservatism (see, [11-14] and the references therein).

The main objective of this paper is to design a filter for discrete T-S fuzzy Fornasini-Marchesini Models with disturbance in FF domain. The aim is to find a filter 2D T-S fuzzy FM model over FF ranges for a given 2D T-S FM such that the associated filtering error meets a prescribed $H_{\infty}$ attenuation level. Then, the sufficient conditions for the existence of the desired filter are derived to ensure the asymptotic stability and FF $H_{\infty}$ performance. A systematic filter design scheme is proposed, which could reduce the conservatism of the results compared to the one considered in EF domain. Finally, a simulation example demonstrates the usefulness of the proposed method.

Notations Superscript " $T$ " stands for matrix transposition. In symmetric block matrices or long matrix expressions, we use an asterisk " $*$ " to represent a term that is induced by symmetry. Notation $P>0$ means that matrix $P$ is positive. $I$ denotes an identity matrix with appropriate dimension. Generally, $\operatorname{sym}\{A\}$ denotes $A+A^{T}$, $\operatorname{diag}\{.$.

\footnotetext{
*e-mail: khaddouj.benmeziane@isga.ma

**e-mail: abderrahim.elamrani@usmba.ac.ma

***e-mail: ismail.boumhidi@usmba.ac.ma
}

stands for block diagonal matrix. The $l_{2}$ norm for a $2 \mathrm{D}$ signal $u(i, j)$ is given by

$$
\|u\|_{2}=\sqrt{\sum_{i=0}^{\infty} \sum_{j=0}^{\infty} u^{T}(i, j) u(i, j)}
$$

where $u(i, j)$ is said to be in the space $l_{2}\{[0, \infty),[0, \infty)\}$ or $l_{2}$, for simplicity, if $\|u\|_{2}<\infty$. A 2D signal $u(i, j)$ in the $l_{2}$ space is an energy-bounded signal.

\section{Problem description and preliminaries}

\subsection{Problem description}

In this paper, we consider a class of 2D nonlinear discretetime systems described by the following T-S FMLSS fuzzy model

Plant Rule l: IF $\theta_{1}(k)$ is $\tilde{N}_{1}^{l}, \theta_{2}(k)$ is $\tilde{N}_{2}^{l}, \ldots \quad$ and $\theta_{\alpha}(k)$ is $\tilde{N}_{\alpha}^{l}$, Then,

$$
\begin{aligned}
x_{i+1, j+1} & =A_{1 l} x_{i, j+1}+A_{2 l} x_{i+1, j}+B_{1 l} u_{i, j+1}+B_{2 l} u_{i+1, j} \\
y_{i, j} & =C_{l} x_{i, j}+D_{l} u_{i, j} \\
z_{i, j} & =E_{l} x_{i, j}
\end{aligned}
$$

where $\left(\tilde{N}_{1}^{l}, \ldots, \tilde{N}_{\alpha}^{l}\right)$ are the fuzzy sets; $l$ is the number of IFTHEN rules $(l=1,2, \ldots, r) ; \theta(k)=\left[\theta_{1}(k), \theta_{2}(k), \ldots, \theta_{\alpha}(k)\right]$ are the premise variables; $k=\{(i, j+1),(i+1, j)\} ; x(i, j) \in$ $\mathbb{R}^{n}$ is the state vector; $y(i, j) \in \mathbb{R}^{n_{y}}$ is the measured output; $z_{i, j} \in \mathbb{R}_{z}^{n}$ is the signal to be estimated; $u(i, j) \in \mathbb{R}^{p}$ is the noise input (that belongs to $\left.l_{2}\{[0, \infty),[0, \infty)\}\right) ;\left(A_{1 l}, B_{1 l}\right.$, $\left.A_{2 l}, B_{2 l}, C_{l}, D_{l}, E_{l}\right)$ are known real matrices with appropriate dimensions. The frequency spectrum of the exogenous noise $u(i, j)$ is assumed to belong to a known rectangular region $\Omega$, where

$$
\begin{aligned}
\Omega \triangleq & \left\{\left(\mu_{1}, \mu_{2}\right) \in \mathbb{R} \mid \mu_{1}^{a} \leq \mu_{1} \leq \mu_{1}^{b} ; \mu_{2}^{a} \leq \mu_{2} \leq \mu_{2}^{b} ;\right. \\
& \left.\mu_{1}^{a}, \mu_{2}^{a}, \mu_{1}^{b}, \mu_{2}^{b} \in[-\pi, \pi]\right\}
\end{aligned}
$$

where $\mu_{1}^{a}, \mu_{1}^{b}, \mu_{2}^{a}$ and $\mu_{2}^{b}$ are known scalars. 
The weighting fuzzy functions are given by

$$
h_{l}(\theta(k))=\frac{\prod_{\eta=1}^{\alpha} \tilde{N}_{l \eta}(\theta(k))}{\sum_{l=1}^{r} \prod_{\eta=1}^{\alpha} \tilde{N}_{l \eta}(\theta(k))}
$$

where $\tilde{N}_{l \eta}\left(\theta_{\eta}(k)\right)$ is the grade of membership of $\theta_{\eta}(k)$ in $\tilde{N}_{l \eta}$. One can see that the fuzzy basis functions satisfy:

$$
\sum_{l=1}^{r} h_{l}(\theta(k))=1, \quad h_{l}(\theta(k)) \in[0,1], \quad l=1, \ldots, r
$$

Via using inference product, singleton fuzzifer and centeraverage defuzzifer, nonlinear system (1) can be described by:

$$
\begin{aligned}
x_{i+1, j+1} & =A_{1}(h) x_{i, j+1}+A_{2}(h) x_{i+1, j} \\
& +B_{1}(h) u_{i, j+1}+B_{2}(h) u_{i+1, j} \\
y_{i, j} & =C(h) x_{i, j}+D(h) u_{i, j} \\
z_{i, j} & =E(h) x_{i, j} \\
x(i, 0) & =x_{i, 0}, \quad x(0, j)=x_{0, j}, i, j \geq 0
\end{aligned}
$$

where

$$
\left[\begin{array}{cc}
A_{1}(h) & B_{1}(h) \\
A_{2}(h) & B_{2}(h) \\
C(h) & D(h) \\
E(h) & 0
\end{array}\right]=\sum_{l=1}^{r} h_{l}(\theta(k))\left[\begin{array}{cc}
A_{1 l} & B_{1 l} \\
A_{2 l} & B_{2 l} \\
C_{l} & D_{l} \\
E_{l} & 0
\end{array}\right]
$$

In order to estimate the signal $z_{i, j}$ a fuzzy Roesser-type filter is designed as follows:

Plant Rule l: IF $\theta_{1}(k)$ is $\tilde{N}_{1}^{l}, \theta_{2}(k)$ is $\tilde{N}_{2}^{l}, \ldots \quad$ and $\theta_{\alpha}(k)$ is $\tilde{N}_{\alpha}^{l}$, Then,

$$
\begin{aligned}
\hat{x}_{i+1, j+1} & =\hat{A}_{1 l} \hat{x}_{i, j+1}+\hat{A}_{2 l} \hat{x}_{i+1, j}+\hat{B}_{1 l} y_{i, j}+\hat{B}_{2 l} y_{i, j} \\
\hat{z}_{i, j} & =\hat{C}_{l} \hat{x}_{i, j}
\end{aligned}
$$

where $\hat{x}_{i, j}$ is the filter state vector; $\hat{z}_{i, j}$ is an estimation of $z_{i, j}$; and $\left(\breve{A}_{1 l}, \breve{A}_{2 l}, \breve{B}_{1 l}, \breve{B}_{2 l}, \breve{C}_{l}\right)$ are the parameters of filter matrices that are appropriately dimensioned real matrices to be determined. lows:

The defuzzified output of system (7) is obtained as fol-

$$
\begin{aligned}
\hat{x}_{i+1, j+1} & =\hat{A}_{1}(h) \hat{x}_{i, j+1}+\hat{A}_{2}(h) x_{i+1, j} \\
& +\hat{B}_{1}(h) y_{i, j}+\hat{B}_{2}(h) y_{i, j} \\
\hat{z}_{i, j} & =\hat{C}(h) \hat{x}_{i, j}
\end{aligned}
$$

where

$$
\left[\begin{array}{cc}
\hat{A}_{1}(h) & \hat{B}_{1}(h) \\
\hat{A}_{2}(h) & \hat{B}_{2}(h) \\
\hat{C}(h) &
\end{array}\right]=\sum_{l=1}^{r} h_{l}(\theta(k))\left[\begin{array}{cc}
\hat{A}_{1 l} & \hat{B}_{1 l} \\
\hat{A}_{2 l} & \hat{B}_{2 l} \\
\hat{C}_{l} &
\end{array}\right]
$$

Defining the augmented state vector $\xi_{i, j}:=\left[\begin{array}{ll}x_{i, j}^{T} & \breve{x}_{i, j}^{T}\end{array}\right]^{T}$, $e_{i, j}=y_{i, j}-\breve{y}_{i, j}$, we can obtain the following error system :

$$
\begin{aligned}
\xi_{i+1, j+1} & =\bar{A}_{1}(h) \xi_{i, j+1}+\bar{A}_{2}(h) \xi_{i+1, j} \\
& +\bar{B}_{1}(h) u_{i, j+1}+\bar{B}_{2}(h) u_{i+1, j} \\
e_{i, j} & =\bar{C}(h) \xi_{i, j} \\
\bar{A}_{1}(h) & =\left[\begin{array}{cc}
A_{1}(h) & 0 \\
\hat{B}_{1}(h) C(h) & \hat{A}_{1}(h)
\end{array}\right] ; \bar{B}_{1}(h)=\left[\begin{array}{c}
B_{1}(h) \\
\hat{B}_{1}(h) D(h)
\end{array}\right] ; \\
\mathcal{A}_{2}(h) & =\left[\begin{array}{cc}
A_{2}(h) & 0 \\
\hat{B}_{2}(h) C(h) & \hat{A}_{2}(h)
\end{array}\right] ; \bar{B}_{2}(h)=\left[\begin{array}{c}
B_{2}(h) \\
\hat{B}_{2}(h) D(h)
\end{array}\right] ; \\
\bar{C}(h) & =\left[\begin{array}{ll}
E(h) & -\hat{C}(h)
\end{array}\right]
\end{aligned}
$$

The problem addressed in this work can be formulated as follows: Given a scalar $\gamma$ and a rectangular FF domain in (3), the filtering error system (10) is said to have a FF gain $\gamma$, if it satisfies the following two conditions:

- Error system (10) is asymptotically stable when $u(i, j) \equiv 0$.

- under the zero boundary condition, the inequality

$$
\sup _{0 \neq u_{i, j} \in l_{2}\{[0, \infty),[0, \infty)\}}\left\|e_{i, j}\right\|_{2} \leq \gamma^{2}\left\|u_{i, j}\right\|_{2}
$$

holds for all solutions of (10) with $u_{i, j} \in$ $l_{2}\{[0, \infty),[0, \infty)\}$ such the following hold

$$
\begin{aligned}
& e^{j \mu_{3}^{b}} \sum_{i=0}^{\infty} \sum_{j=0}^{\infty}\left[\left(\xi_{i+1, j+1}-e^{j \mu{ }_{1}^{a}} \xi_{i, j+1}\right)\right. \\
& \left.\left(\xi_{i+1, j+1}-e^{j \mu_{1}^{b}} \xi_{i, j+1}\right)^{T}\right] \leq 0 \\
& e^{j \mu_{4}^{b}} \sum_{i=0}^{\infty} \sum_{j=0}^{\infty}\left[\left(\xi_{i+1, j+1}-e^{j \mu_{2}^{a}} \xi_{i+1, j}\right)\right. \\
& \left.\left(\xi_{i+1, j+1}-e^{j \mu_{2}^{b}} \xi_{i+1, j}\right)^{T}\right] \leq 0
\end{aligned}
$$

where $\mu_{3}^{b}=\frac{\mu_{1}^{b}-\mu_{1}^{a}}{2} ; \quad \mu_{4}^{b}=\frac{\mu_{2}^{b}-\mu_{2}^{a}}{2}$

\subsection{Preliminaries}

We introduce the following technical lemmas that are useful for deriving our results.

Lemma 1 [15] From (14), we can obtain (15)

$$
\begin{aligned}
& {\left[\begin{array}{cc}
T+M U+U^{T} M^{T} & * \\
-M^{T}+G U & V-G-G^{T}
\end{array}\right]<0} \\
& T+U^{T} V U<0
\end{aligned}
$$

Lemma 2 [13] Let $\gamma>0$ be a given scalar and a rectangular FF domain (2). For error system (10) is asymptotically stable, $H_{\infty}$ performance (12) is satisfied, if there exist Hermitian $P_{1}, P_{2}, 0<Q_{1}, 0<Q_{2} \in \mathbb{H}_{2 n}$, such that

$$
\begin{array}{r}
{\left[\begin{array}{cc}
\mathcal{A}(h) & \mathcal{B}(h) \\
I & 0
\end{array}\right]^{T}\left[\begin{array}{cc}
P & \Lambda^{*} Q \\
Q \Lambda & -\mathcal{R}
\end{array}\right]\left[\begin{array}{cc}
\mathcal{A}(h) & \mathcal{B}(h) \\
I & 0
\end{array}\right]} \\
+\left[\begin{array}{cc}
\mathcal{C}(h)^{T} \mathcal{C}(h) & \mathcal{C}(h)^{T} \mathcal{D}(h) \\
\mathcal{D}(h)^{T} \mathcal{C}(h) & -\gamma^{2} I+\mathcal{D}(h)^{T} \mathcal{D}(h)
\end{array}\right]<0
\end{array}
$$

where

$$
\begin{aligned}
\mathcal{A}(h) & =\left[\begin{array}{cc}
\bar{A}_{1}(h) & \bar{A}_{2}(h)
\end{array}\right] ; \mathcal{B}(h)=\left[\begin{array}{cc}
\bar{B}_{1}(h) & \bar{B}_{2}(h)
\end{array}\right] ; \\
C(h) & =\left[\begin{array}{cc}
\bar{C}(h) & 0 \\
0 & \bar{C}(h)
\end{array}\right] ; \mathcal{D}(h)=\left[\begin{array}{cc}
\bar{D}(h) & 0 \\
0 & \bar{D}(h)
\end{array}\right] ; \\
Q & =\left[\begin{array}{cc}
Q_{1} & Q_{2}
\end{array}\right] ; P=P_{1}+P_{2} ; \\
\Lambda & =\left[\begin{array}{cc}
e^{-j \mu_{3}^{a} I_{2 \bar{n}}} & 0 \\
0 & e^{-j \mu_{4}^{a} I_{2 \bar{n}}}
\end{array}\right] ; \\
\mathcal{R} & =\left[\begin{array}{cc}
P_{1}+2 \cos \left(\mu_{3}^{b}\right) Q_{1} & 0 \\
0 & P_{2}+2 \cos \left(\mu_{4}^{b}\right) Q_{2}
\end{array}\right] ; \\
\mu_{3}^{a} & =\frac{\mu_{1}^{a}+\mu_{1}^{b}}{2}, \quad \mu_{4}^{a}=\frac{\mu_{2}^{a}+\mu_{2}^{b}}{2} ; \\
\mu_{3}^{b} & =\frac{\mu_{1}^{b}-\mu_{1}^{a}}{2}, \quad \mu_{4}^{b}=\frac{\mu_{2}^{b}-\mu_{2}^{a}}{2}
\end{aligned}
$$




\section{Main results}

\subsection{FF performance analysis}

On the basis of Lemmas 1 and 2, we give the following theorem, which can guarantee the asymptotical stability and the $H_{\infty}$ performance of error system (10) in the FF domain of input noise.

Theorem 1 Let $\gamma>0$ be a given scalar and a rectangular FF domain (2), a filter of form (8) exists such that the filtering error system in (10) is asymptotically stable and $H_{\infty}$ performance (12) is satisfied, if and only if there exist Hermitian matrices $P=P_{1}+P_{2}, Q=\left[\begin{array}{ll}Q_{1} & Q_{2}\end{array}\right]$, symmetric matrices $W_{1}, W_{2}$ and matrices $M_{1}, M_{2}, G_{1}, G_{2}, F_{1}$ and $H$ satisfying $Q_{1}>0, Q_{2}>0, W_{1}>0, W_{2}>0$ and

$$
\begin{aligned}
& \Phi=\left[\begin{array}{ccccc}
\Phi_{11} & \Phi_{12} & \Phi_{13} & \mathcal{A}^{T}(h) G_{2}^{T} & C^{T} \\
* & \Phi_{22} & \Phi_{23} & \mathcal{B}^{T}(h) G_{2}^{T} & \mathcal{D}^{T} \\
* & * & \Phi_{33} & \Lambda^{*} Q-G_{2}^{T} & 0 \\
* & * & * & -\mathcal{R} & 0 \\
* & * & * & * & -I
\end{array}\right]<0 \\
& \Upsilon=\left[\begin{array}{ccc}
\Upsilon_{11} & F_{1} \bar{A}_{2}(h) & -F_{1}+\bar{A}_{1}^{T}(h)^{T} H^{T} \\
* & -W_{2} & \bar{A}_{2}^{T}(h)^{T} H^{T} \\
* & * & W_{1}+W_{2}-\operatorname{sym}(H)
\end{array}\right]<0
\end{aligned}
$$

$$
\begin{aligned}
\Phi_{11} & =M_{1} \mathcal{A}(h)+\mathcal{A}^{T}(h) M_{1}^{T} ; \\
\Phi_{12} & =M_{1} \mathcal{B}(h)+\mathcal{A}^{T}(h) M_{2}^{T} ; \\
\Phi_{13} & =\mathcal{A}^{T}(h) G_{1}^{T}-M_{1} ; \\
\Phi_{22} & =-\gamma^{2} I+\operatorname{sym}\left[M_{2} \mathcal{B}(h)\right] ; \\
\Phi_{23} & =\mathcal{B}^{T}(h) G_{1}^{T}-M_{2} ; \\
\Phi_{33} & =P-G_{1}-G_{1}^{T} ; \\
\Upsilon_{11} & =-W_{1}+\operatorname{sym}\left(F_{1} \bar{A}_{1}(h)\right)
\end{aligned}
$$

Proof 1 First, we prove that (16) is equivalent to (18). Condition (16) can be rewritten as

$$
T+U^{T} V U<0
$$

Where

$$
\begin{aligned}
U & =\left[\begin{array}{cc}
\mathcal{A}(h) & \mathcal{B}(h) \\
I & 0
\end{array}\right] ; \quad V=\left[\begin{array}{cc}
P & \Lambda^{*} Q \\
Q \Lambda & -\mathcal{R}
\end{array}\right] ; \\
T & =\left[\begin{array}{cc}
\mathcal{C}(h)^{T} \mathcal{C}(h) & C(h)^{T} \mathcal{D}(h) \\
\mathcal{D}(h)^{T} C(h) & -\gamma^{2} I+\mathcal{D}(h)^{T} \mathcal{D}(h)
\end{array}\right]
\end{aligned}
$$

At this stand, by using Lemma 2, (20) is equivalent to

$$
\left[\begin{array}{cc}
T+M U+U^{T} M^{T} & U^{T} G^{T}-M \\
-M^{T}+G U & V-G-G^{T}
\end{array}\right]<0
$$

We chose $M$ and $G$ are expressed as the following structures:

$$
M=\left[\begin{array}{cc}
M_{1} & 0 \\
M_{2} & 0
\end{array}\right] ; \quad G=\left[\begin{array}{cc}
G_{1} & 0 \\
G_{2} & 0
\end{array}\right]
$$

which, using Schur complement, leads to given (18). Second step, let us construct a Lyapunov function inequality, $\bar{A}_{1}(h)$ and $\bar{A}_{2}(h)$ is stable if and only if there exist symmetric matrices $W_{1}>0, W_{2}>0$ such that

$$
\begin{aligned}
& {\left[\begin{array}{cc}
\bar{A}_{1}(h) & \bar{A}_{2}(h)
\end{array}\right]^{T}\left(W_{1}+W_{2}\right)\left[\begin{array}{cc}
\bar{A}_{1}(h) & \bar{A}_{2}(h)
\end{array}\right] } \\
- & {\left[\begin{array}{cc}
W_{1} & 0 \\
0 & W_{2}
\end{array}\right]<0 }
\end{aligned}
$$

which is rewritten in the form

$$
\hat{T}+\hat{U}^{T} \hat{V} \hat{U}<0
$$

Where

$$
\begin{aligned}
\hat{T} & =\left[\begin{array}{cc}
-W_{1} & 0 \\
0 & -W_{2}
\end{array}\right] ; \hat{V}=W_{1}+W_{2} ; \\
\hat{U} & =\mathcal{A}(h)=\left[\begin{array}{ll}
\bar{A}_{1}(h) & \bar{A}_{2}(h)
\end{array}\right] ;
\end{aligned}
$$

We chose $F$ follows.

$$
F=\left[\begin{array}{ll}
F_{1} & 0
\end{array}\right]^{T}
$$

Using Lemma 1, (25-26) are equivalent to (19).

\subsection{Fuzzy Filter Design}

Theorem 1 fails to provide explicit solutions to filter realization. On the basis of Theorem 2, we are trying to find methods for parameterizing fuzzy filters for the discrete T-S fuzzy system (1). Via specifying the structure of the slack matrices in Theorem 2, we can derive the following result.

Theorem 2 Let $\gamma>0$ be a given scalar and a rectangular FF domain (2), a filter of form (8) exists such that the filtering error system in (10) is asymptotically stable and $H_{\infty}$ performance (12) is satisfied, if there exist matrices $\hat{A}_{1 i}, \hat{B}_{1 i}, \hat{A}_{2 i}, \hat{B}_{2 i}, \hat{C}_{1 i}, \hat{D}_{1 i}, M_{1 u}, G_{2 u}, M_{2 t}, G_{1 t}, H_{1 t}, F_{1 t}, V$, $u=1,2,3,4, t=1,2, P_{1 s}, Q_{1 s}>0, W_{1 s}>0, P_{2 s}, Q_{2 s}>0$, $W_{2 s}>0, s=1,2,3$, satisfying

$$
\tilde{\Psi}=\left[\begin{array}{c|c}
\tilde{\Psi}_{1} & \tilde{\Psi}_{2} \\
\hline * & \tilde{\Psi}_{3}
\end{array}\right]<0
$$

$$
\tilde{\Upsilon}=\left[\begin{array}{cccccc}
\tilde{\Upsilon}_{11} & \tilde{\Upsilon}_{12} & \tilde{\Upsilon}_{13} & E \hat{A}_{2 i} & \tilde{\Upsilon}_{15} & \tilde{\Upsilon}_{16} \\
* & \tilde{\Upsilon}_{22} & \tilde{\Upsilon}_{23} & \hat{A}_{2 i}^{T} & \tilde{\Upsilon}_{25} & \tilde{\Upsilon}_{26} \\
* & * & -W_{21} & -W_{22} & \tilde{\Upsilon}_{35} & A_{2 j}^{T} H_{12}^{T} \\
* & * & * & -W_{23} & \tilde{\Upsilon}_{45} & \hat{A}_{2 i}^{T} \\
* & * & * & * & \tilde{\Upsilon}_{55} & \tilde{\Upsilon}_{56} \\
* & * & * & * & * & \tilde{\Upsilon}_{66}
\end{array}\right]<0
$$

$$
\tilde{\Psi}_{1}=\left[\begin{array}{cccccc}
\tilde{\Psi}_{1_{11}} & \tilde{\Psi}_{1_{12}} & \tilde{\Psi}_{1_{13}} & \tilde{\Psi}_{1_{14}} & \tilde{\Psi}_{1_{15}} & \tilde{\Psi}_{1_{16}} \\
* & \tilde{\Psi}_{1_{22}} & M_{12} A_{2 j} & \operatorname{sym}\left[\hat{A}_{2 i}\right] & \tilde{\Psi}_{1_{25}} & \tilde{\Psi}_{1_{26}} \\
* & * & \tilde{\Psi}_{1_{33}} & A_{2 j}^{T} M_{14}^{T} & \tilde{\Psi}_{1_{35}} & \tilde{\Psi}_{1_{36}} \\
* & * & * & \tilde{\Psi}_{1_{44}} & \tilde{\Psi}_{1_{45}} & \tilde{\Psi}_{1_{46}} \\
* & * & * & * & \tilde{\Psi}_{1_{55}} & \tilde{\Psi}_{1_{56}} \\
* & * & * & * & * & \tilde{\Psi}_{1_{66}}
\end{array}\right] ;
$$

$$
\begin{aligned}
\tilde{\Psi}_{2} & =\left[\begin{array}{cccccccc}
\tilde{\Psi}_{11} & \tilde{\Psi}_{12} & \tilde{\Psi}_{13} & \tilde{\Psi}_{14} & \tilde{\Psi}_{15} & \tilde{\Psi}_{16} & \tilde{\Psi}_{17} & 0 \\
\tilde{\Psi}_{21} & \tilde{\Psi}_{22} & \tilde{\Psi}_{23} & \tilde{\Psi}_{24} & 0 & \tilde{\Psi}_{26} & 0 & \tilde{\Psi}_{88} \\
\tilde{\Psi}_{31} & \tilde{\Psi}_{32} & \tilde{\Psi}_{33} & \tilde{\Psi}_{34} & \tilde{\Psi}_{35} & \tilde{\Psi}_{36} & \tilde{\Psi}_{37} & 0 \\
\tilde{\Psi}_{41} & \tilde{\Psi}_{42} & \tilde{\Psi}_{43} & \tilde{\Psi}_{44} & 0 & \tilde{\Psi}_{46} & 0 & -\hat{C}_{i}^{T} \\
\tilde{\Psi}_{51} & \tilde{\Psi}_{52} & \tilde{\Psi}_{53} & \tilde{\Psi}_{54} & \tilde{\Psi}_{55} & \tilde{\Psi}_{56} & \tilde{\Psi}_{57} & 0 \\
\tilde{\Psi}_{61} & \tilde{\Psi}_{62} & \tilde{\Psi}_{63} & \tilde{\Psi}_{64} & \tilde{\Psi}_{65} & \tilde{\Psi}_{66} & 0 & \tilde{\Psi}_{88}
\end{array}\right] ; \\
\tilde{\Psi}_{3} & =\left[\begin{array}{cccccccc}
\tilde{\Psi}_{311} & \tilde{\Psi}_{312} & \tilde{\Psi}_{313} & \tilde{\Psi}_{314} & \tilde{\Psi}_{315} & \tilde{\Psi}_{316} & 0 & 0 \\
* & \tilde{\Psi}_{322} & \tilde{\Psi}_{323} & \tilde{\Psi}_{324} & \tilde{\Psi}_{325} & \tilde{\Psi}_{326} & 0 & 0 \\
* & * & \tilde{\Psi}_{33} & \tilde{\Psi}_{334} & 0 & 0 & 0 & 0 \\
* & * & * & \tilde{\Psi}_{344} & 0 & 0 & 0 & 0 \\
* & * & * & * & \tilde{\Psi}_{355} & \tilde{\Psi}_{356} & 0 & 0 \\
* & * & * & * & * & \tilde{\Psi}_{366} & 0 & 0 \\
* & * & * & * & * & * & -I & 0 \\
* & * & * & * & * & * & * & -I
\end{array}\right]
\end{aligned}
$$




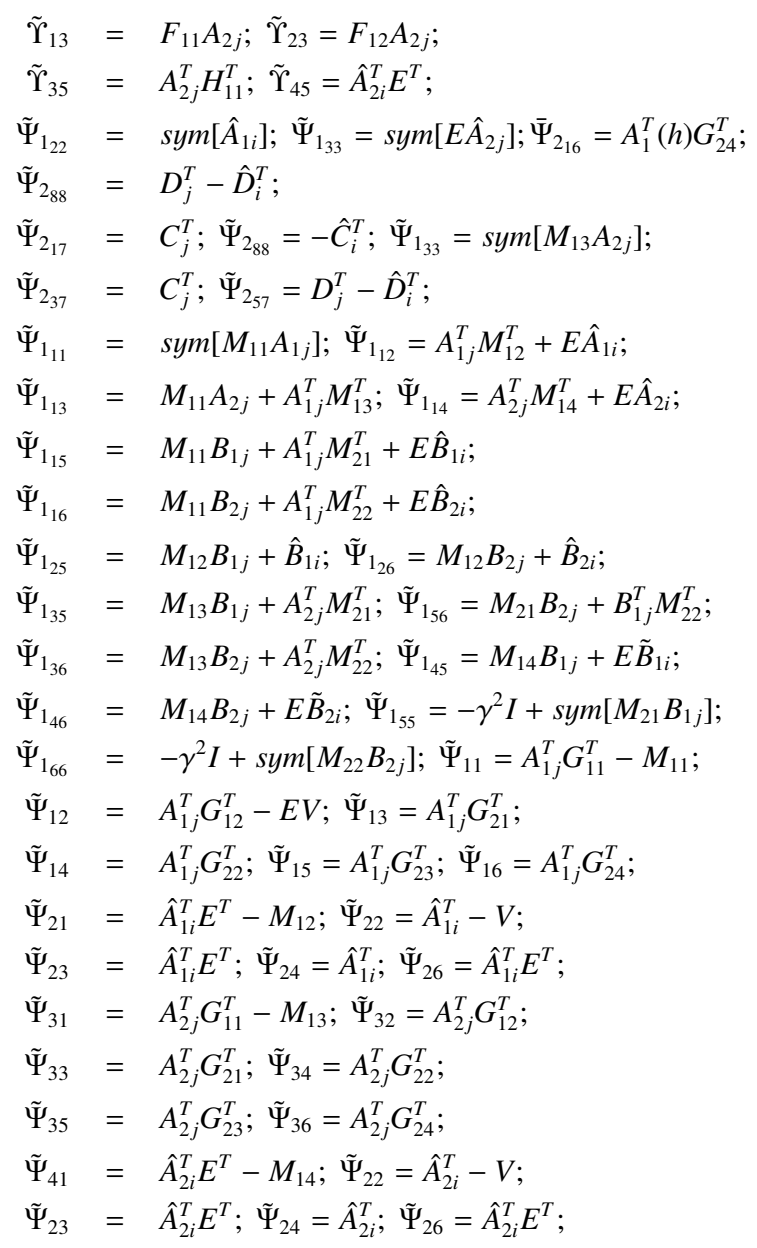

$\tilde{\Psi}_{51}=B_{1 j}^{T} G_{11}^{T}+\hat{B}_{1 i}^{T} E^{T}-M_{21} ; \tilde{\Psi}_{52}=B_{1 j}^{T} G_{12}^{T}+\hat{B}_{1 i}^{T} ;$

$\tilde{\Psi}_{53}=B_{1 j}^{T} G_{21}^{T}+\hat{B}_{1 i}^{T} E^{T} ; \tilde{\Psi}_{54}=B_{1 j}^{T} G_{22}^{T}+\hat{B}_{1 i}^{T} ;$

$\tilde{\Psi}_{55}=B_{1 j}^{T} G_{23}^{T} ; \tilde{\Psi}_{56}=B_{1 j}^{T} G_{24}^{T}+\hat{B}_{1 i}^{T} E^{T} ;$

$\tilde{\Psi}_{61}=B_{2 j}^{T} G_{11}^{T}-M_{22} ; \tilde{\Psi}_{62}=B_{2 j}^{T} G_{12}^{T}+\hat{B}_{2 i}^{T} ;$

$\tilde{\Psi}_{63}=B_{2 j}^{T} G_{21}^{T}+\hat{B}_{2 i}^{T} E^{T} ; \tilde{\Psi}_{64}=B_{2 j}^{T} G_{22}^{T}+\hat{B}_{2 i}^{T} ;$

$\tilde{\Psi}_{65}=B_{2 j}^{T} G_{23}^{T} ; \tilde{\Psi}_{66}=B_{2 j}^{T} G_{24}^{T}+\hat{B}_{2 i}^{T} E^{T} ;$

$\tilde{\Psi}_{3_{11}}=P_{11}+P_{21}-G_{11}-G_{11}^{T} ; \tilde{\Psi}_{313}=e^{-j \mu_{3}^{a}} Q_{11}-G_{21}^{T} ;$

$\tilde{\Psi}_{3_{12}}=P_{12}+P_{22}-E V-G_{21}^{T} ; \tilde{\Psi}_{3_{14}}=e^{-j \mu_{3}^{a}} Q_{12}-G_{22}^{T}$;

$\tilde{\Psi}_{315}=e^{-j \mu_{3}^{a}} Q_{21}-G_{23}^{T} ; \tilde{\Psi}_{316}=e^{-j \mu_{3}^{a}} Q_{22}-G_{24}^{T} ;$

$\tilde{\Psi}_{322}=P_{13}+P_{23}-V-V^{T} ; \tilde{\Psi}_{323}=e^{-j \mu_{4}^{a}} Q_{21}^{T}-V^{T} E^{T}$;

$\tilde{\Psi}_{324}=e^{-j \mu_{4}^{a}} Q_{13}-V^{T} ; \tilde{\Psi}_{325}=e^{-j \mu_{4}^{a}} Q_{22}^{T} ;$

$\tilde{\Psi}_{326}=e^{-j \mu_{4}^{a}} Q_{23}-V^{T} E^{T} ; \tilde{\Psi}_{33}=P_{11}-2 \cos \left(\mu_{3}^{b}\right) Q_{11} ;$

$\tilde{\Psi}_{344}=P_{12}-2 \cos \left(\mu_{3}^{b}\right) Q_{12} ; \tilde{\Psi}_{344}=P_{13}-2 \cos \left(\mu_{3}^{b}\right) Q_{13} ;$

$\tilde{\Psi}_{355}=P_{21}-2 \cos \left(\mu_{3}^{b}\right) Q_{21} ; \tilde{\Psi}_{356}=P_{22}-2 \cos \left(\mu_{3}^{b}\right) Q_{22} ;$

$\tilde{\Psi}_{366}=P_{23}-2 \cos \left(\mu_{3}^{b}\right) Q_{23} ; \tilde{\Upsilon}_{12}=-W_{12}+A_{1 j}^{T} F_{12}^{T}+E \hat{A}_{1 i} ;$

$\tilde{\Upsilon}_{11}=-W_{11}+\operatorname{sym}\left(F_{11} A_{1 j}\right)$;

$\tilde{\Upsilon}_{22}=-W_{13}+\operatorname{sym}\left(\hat{A}_{1 i}\right) ; \tilde{\Upsilon}_{15}=-F_{11}+A_{1 j}^{T} H_{11}^{T} ;$

$\tilde{\Upsilon}_{16}=-E V+A_{1 j}^{T} H_{12}^{T} ; \tilde{\Upsilon}_{25}=-F_{12}+\hat{A}_{1 i}^{T} E^{T} ;$

$\tilde{\Upsilon}_{26}=\hat{A}_{1 i}^{T}-V ; \tilde{\Upsilon}_{55}=W_{11}+W_{21}-\operatorname{sym}\left(H_{11}\right) ;$

$\tilde{\Upsilon}_{56}=W_{12}+W_{22}-E V-H_{12}^{T}$;

$\tilde{\Upsilon}_{66}=W_{13}+W_{23}-\operatorname{sym}(V) ; E=\left[\begin{array}{ll}I & 0\end{array}\right]^{T}$
Moreover, under the above conditions, we can obtain a state-space realization of filter (5) with the following parameters as

$$
\begin{aligned}
\hat{A}_{1 i} & =V^{-1} \breve{A}_{1 i} ; \hat{A}_{2 i}=V^{-1} \breve{A}_{2 i} ; \\
\hat{B}_{1 i} & =V^{-1} \breve{B}_{1 i} ; \hat{B}_{2 i}=V^{-1} \breve{B}_{2 i} ; \\
\hat{C}_{i} & =\breve{C}_{i} .
\end{aligned}
$$

Proof 2 Parameterise slack matrices $M_{1}, M_{2}, G_{1}, G_{2}, F_{1}$ and $H$ in Theorem 1 as

$$
\begin{aligned}
M_{1} & =\left[\begin{array}{ll}
M_{11} & V \\
M_{12} & V \\
M_{13} & 0 \\
M_{14} & V
\end{array}\right] ; \quad G_{2}=\left[\begin{array}{ll}
G_{21} & V \\
G_{22} & V \\
G_{23} & 0 \\
G_{24} & V
\end{array}\right] ; \\
M_{2} & =\left[\begin{array}{ll}
M_{21} & 0 \\
M_{22} & 0
\end{array}\right] ; \quad G_{1}=\left[\begin{array}{ll}
G_{11} & V \\
G_{12} & V
\end{array}\right] ; \\
F & =\left[\begin{array}{ll}
F_{11} & V \\
F_{12} & V
\end{array}\right], H=\left[\begin{array}{ll}
H_{11} & V \\
H_{12} & V
\end{array}\right]
\end{aligned}
$$

Moreover, for matrix variables $P_{1}, Q_{1}>0, P_{2}$, $Q_{2}>0, W_{1}>0, W_{2}>0$ in theorem 1 , we define:

$$
\begin{aligned}
& P_{1}=\left[\begin{array}{cc}
P_{11} & P_{12} \\
* & P_{13}
\end{array}\right] ; P_{2}=\left[\begin{array}{cc}
P_{21} & P_{22} \\
* & P_{23}
\end{array}\right] ; \\
& Q_{1}=\left[\begin{array}{cc}
Q_{11} & Q_{12} \\
* & Q_{13}
\end{array}\right] ; Q_{2}=\left[\begin{array}{cc}
Q_{21} & Q_{22} \\
* & Q_{23}
\end{array}\right] ; \\
& W_{1}=\left[\begin{array}{cc}
W_{11} & W_{12} \\
* & W_{13}
\end{array}\right] ; W_{2}=\left[\begin{array}{cc}
W_{21} & W_{22} \\
* & W_{23}
\end{array}\right]
\end{aligned}
$$

In addition, by replacing (11), (17) and into (18) and (19), and combining (31), (33), we obtain theorem ??, where

$$
\begin{aligned}
& \breve{A}_{1}(h)=V \hat{A}_{1}(h), \breve{A}_{2}(h)=V \hat{A}_{2}(h), \\
& \breve{B}_{1}(h)=V \hat{B}_{1}(h), \breve{B}_{2}(h)=V \hat{B}_{2}(h),
\end{aligned}
$$

\section{Numerical Example}

Consider a 2D discrete-time T-S fuzzy system with two rules, given by [13]

Plant Rule 1: $\operatorname{IF} \theta_{1}(k)$ is $\tilde{N}_{1}^{1}, \theta_{2}(k)$ is $\tilde{N}_{2}^{1}$, Then,

$$
\begin{aligned}
x_{i+1, j+1} & =A_{11} x_{i, j+1}+A_{21} x_{i+1, j}+B_{11} u_{i, j+1}+B_{21} u_{i+1, j} \\
y_{i, j} & =C_{1} x_{i, j}+D_{1} u_{i, j} \\
z_{i, j} & =E_{1} x_{i, j}
\end{aligned}
$$

Plant Rule 2: IF $\theta_{1}(k)$ is $\tilde{N}_{1}^{2}, \theta_{2}(k)$ is $\tilde{N}_{2}^{2}$, Then,

$$
\begin{aligned}
x_{i+1, j+1} & =A_{12} x_{i, j+1}+A_{22} x_{i+1, j}+B_{12} u_{i, j+1}+B_{22} u_{i+1, j} \\
y_{i, j} & =C_{2} x_{i, j}+D_{2} u_{i, j} \\
z_{i, j} & =E_{1} x_{i, j}
\end{aligned}
$$

where

$$
\begin{aligned}
& A_{11}=\left[\begin{array}{cc}
0.1 & -0.2 \\
-0.2 & 0.1
\end{array}\right] A_{21}=\left[\begin{array}{cc}
0.25 & 0.1 \\
-0.05 & 0.3
\end{array}\right] ; \\
& A_{12}=\left[\begin{array}{cc}
0.3 & -0.2 \\
-0.2 & 0.3
\end{array}\right] A_{22}=\left[\begin{array}{cc}
0.25 & 0.1 \\
-0.05 & 0.5
\end{array}\right] ; \\
& B_{11}=\left[\begin{array}{c}
0.1 \\
0.25
\end{array}\right] ; B_{12}=\left[\begin{array}{c}
0.1 \\
0.4
\end{array}\right] ; D_{1}=D_{2}=0.1 ; \\
& B_{21}=\left[\begin{array}{c}
0 \\
0.28
\end{array}\right] ; B_{22}=\left[\begin{array}{c}
0 \\
0.2
\end{array}\right] ; E_{1}=E_{2}=0.1 ; \\
& C_{1}=C_{2}=\left[\begin{array}{ll}
0 & 1
\end{array}\right]
\end{aligned}
$$


The normalized membership function :

$$
\begin{aligned}
& h_{1}\left(\theta_{i, j}\right)=1-\frac{1}{1+\exp \left(-2\left(\theta_{i, j}-3\right)\right)} \\
& h_{2}\left(\theta_{i, j}\right)=\frac{1}{1+\exp \left(-2\left(\theta_{i, j}-3\right)\right)}
\end{aligned}
$$

The aim is to design a fuzzy discrete filter in the form of (8) to guarantee the asymptotic stability of the filtering error system. Suppose that the FF domain of disturbance input signal is $\left[\frac{\pi}{8}, \frac{\pi}{4}\right] \times\left[\frac{\pi}{8}, \frac{\pi}{4}\right]$. Via using Theorem 4 , the obtained matrix parameters of FF $H_{\infty}$ filter are the following

$$
\begin{aligned}
\hat{A}_{11} & =\left[\begin{array}{ll}
0.1454 & -0.1092 \\
0.0638 & -0.2298
\end{array}\right] ; \breve{B}_{11}=\left[\begin{array}{c}
-0.3219 \\
0.0002
\end{array}\right] ; \\
\breve{A}_{12} & =\left[\begin{array}{ll}
0.2454 & -0.0738 \\
0.0010 & -0.0974
\end{array}\right] ; \breve{B}_{21}=\left[\begin{array}{c}
-0.0923 \\
-0.1105
\end{array}\right] ; \\
\breve{C}_{1} & =\left[\begin{array}{ll}
-1.9560 & 0.4749
\end{array}\right] ; \\
\breve{A}_{21} & =\left[\begin{array}{cc}
-0.1527 & 0.7145 \\
0.0305 & -0.2105
\end{array}\right] ; \breve{B}_{12}=\left[\begin{array}{c}
-0.1504 \\
0.0204
\end{array}\right] ; \\
\breve{C}_{1} & =\left[\begin{array}{ll}
-1.9560 & 0.4749
\end{array}\right] ; \\
\breve{A}_{22} & =\left[\begin{array}{ll}
-0.0101 & 0.8745 \\
0.0174 & -0.0202
\end{array}\right] ; \breve{B}_{22}=\left[\begin{array}{c}
-0.0017 \\
0.0201
\end{array}\right] ; \\
\breve{C}_{2} & =\left[\begin{array}{ll}
-1.9560 & 0.4749
\end{array}\right] ;
\end{aligned}
$$

LMIs (28) and (29) were solved using Yalmip ([16]) and SeDuMi ([17]) in MATLAB 7.6. The comparison result with the technique proposed in Theorem 2 shown in Table 1 , which shows the smaller conservativeness of the approach proposed in this paper.

\begin{tabular}{l|c|c}
\hline Frequency & Methods & $\gamma$ \\
\hline$[0, \pi] \times[0, \pi]$ & TH 6 in $[18]$ & $\mathbf{1 . 7 3 0 2}$ \\
\hline$[0, \pi] \times[0, \pi]$ & TH $3.4(\mathrm{Q}=0)$ in $[13]$ & Inf \\
\hline$[0, \pi] \times[0, \pi]$ & TH 2 $(\mathbf{Q}=\mathbf{0})$ & $\mathbf{0 . 6 3 2 1}$ \\
\hline$\left[\frac{\pi}{8}, \frac{\pi}{4}\right] \times\left[\frac{\pi}{8}, \frac{\pi}{4}\right]$ & Theorem 3.4 in $[13]$ & $\mathbf{0 . 6 0 0 0}$ \\
\hline$\left[\frac{\pi}{8}, \frac{\pi}{4}\right] \times\left[\frac{\pi}{8}, \frac{\pi}{4}\right]$ & TH 2 & $\mathbf{0 . 2 3 5 7}$ \\
\hline
\end{tabular}

Table 1: Comparison of filtering performance obtained in different methods.

Furthermore, under zero boundary conditions, and let the disturbance input be the following form

$$
u\left(e^{j \mu_{1}}, e^{j \mu_{2}}\right)=\left\{\begin{array}{cr}
0.4, & \frac{\pi}{3} \leq \mu_{1}, \mu_{2} \leq \frac{5 \pi}{7} \\
0, & \text { otherwise. }
\end{array}\right\}
$$

Figures 1-3 show the trajectories of states filters vectors $\hat{x}_{1}, \hat{x}_{2}$ and filtering error system of $e_{i, j}$, respectively, it is clear that effectively, the $2 \mathrm{D}$ system is asymptotically stable and converges towards zero. From Figures 1-3, the asymptotic stability of the error system can be clearly observed, while under the zero boundary conditions and the disturbance input (38), we can work out $\frac{\left\|e_{i, j}\right\|_{2}}{\left\|u_{i, j}\right\|_{2}}=0.2205$. So, the condition (9) is satisfied, which means that the error filtering system has a prescribed FF $H_{\infty}$ gain $\gamma=0.2357$.

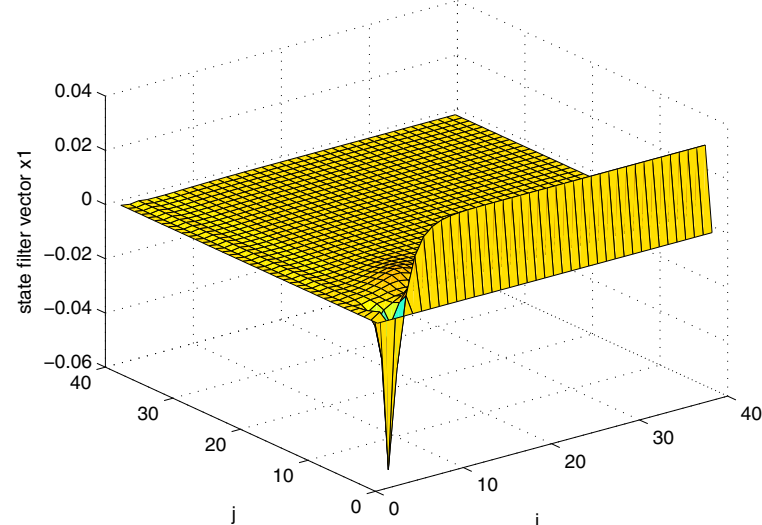

Figure 1. Trajectory of State filter vectors $\hat{x}_{1}$.

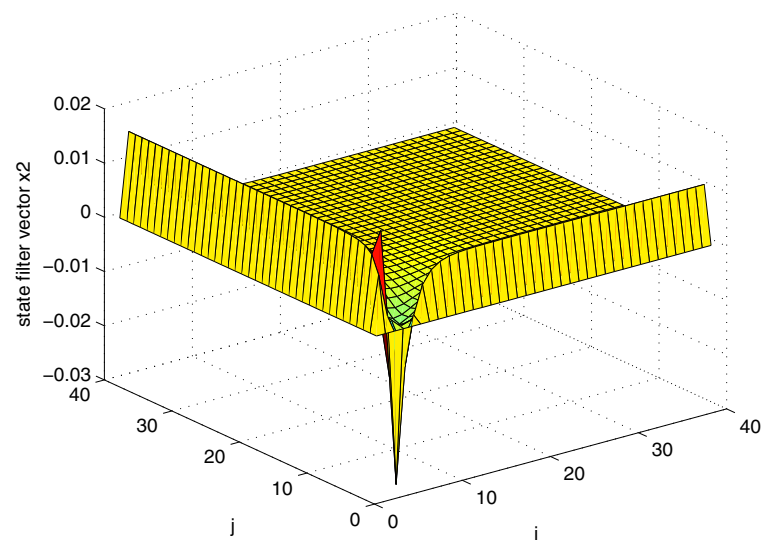

Figure 2. Trajectory of State filter vectors $\hat{x}_{2}$.

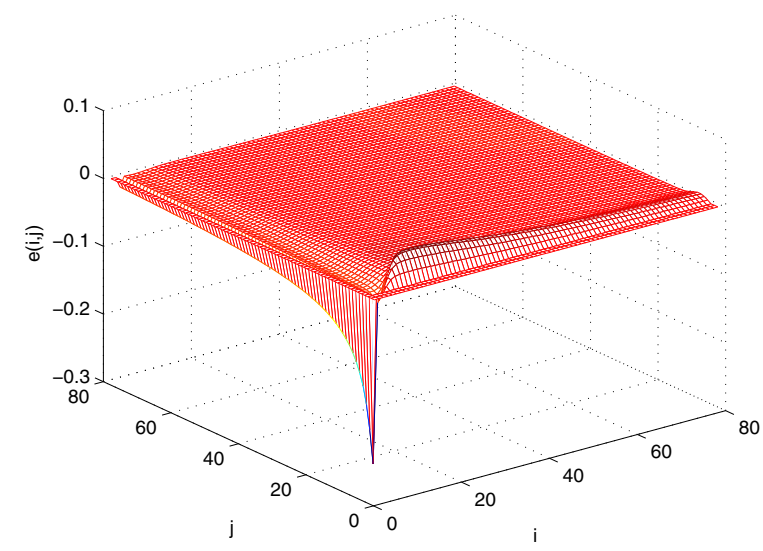

Figure 3. Filtering error response of $e_{i, j}$. 


\section{Conclusion}

This paper has concerned with the problem of the filter design for discrete T-S fuzzy systems in FMLSS model with FF disturbances. Assuming the disturbances is dominated in a known FF domain, a fuzzy-filtering method is proposed to reduce the design conservatism by making use of the frequency information of disturbances. A simulation example has been given to illustrate the effectiveness of the proposed method.

\section{References}

[1] M.-S. Branicky, V.-S. Borkar, S.-K. Mitter, "A unified framework for hybrid control: Model and optimal control theory", IEEE Transactions on Automatic Control, 1998, vol. 43, no. 1, pp. 31-45, 1998.

[2] T. Kaczorek, Ed. "Two-Dimensional Linear Systems". Lecture Notes in Control and Information Science, New York: Springer-Verlag, vol. 68, 1985.

[3] C. Du and L. Xie. " $H_{\infty}$ Control and Filtering of Twodimensional Systems". Springer Verlag, vol. 278, 2002.

[4] X. Li, J. Lam, H. Gao, Y. Gu, "A frequencypartitioning approach to stability analysis of twodimensional discrete systems". Multidimensional Systems and Signal Processing, vol. 26, no. 1, pp. 67-93, 2013.

[5] H. Gao, X. Meng, T. Chen. "New Design of Robust $H_{\infty}$ Filters for 2-D Systems". IEEE Signal Processing Letters, vol. 15, pp. 217-220, 2008.

[6] H. Gao, J. Lam, C. Wang, \& S. Xu. " $H_{\infty}$ model reduction for uncertain two-dimensional discrete systems". Optimal Control Applications and Methods, vol. 26, no. 4, pp. 199-227, 2005.

[7] C. Lin, Q.-G. Wang, T. H. Lee, B. Chen, " $H_{\infty}$ filter design for nonlinear systems with time-delay through T-S fuzzy model approach", IEEE Transactions on Fuzzy Systems, vol. 16, no. 3, pp. 739-746. 2008.

[8] E. Tian, D. Yue, "Reliable $H_{\infty}$ filter design for T$S$ fuzzy model-based networked control systems with random failure", International Journal of Robust and Nonlinear Control, vol. 23, no. 1, pp. 15-32. 2013.
[9] Y. Luo, Z. Wang, J. Liang , G. Wei, F. E. Alsaadi, " $H_{\infty}$ control for 2-D fuzzy systems with interval time-varying delays and missing measurements", IEEE Transactions on Cybernetics, vol. 47, no. 2, pp. 365377. 2017.

[10] H. Nobahari, S. M. Zandavi, H. Mohammadkarimi, "Simplex filter: A novel heuristic filter for nonlinear systems state estimation", Applied Soft Computing, vol. 49, pp. 474-484. 2016.

[11] X. Li, H. Gao. "Robust finite frequency image filtering for uncertain 2-D systems: The FM model case". Automatica, vol. 29, no. 8, pp. 2446-2452, 2013.

[12] A. El-Amrani, B. Boukili, A. Hmamed, A. El Hajjaji and I. Boumhidi. "Robust $H_{\infty}$ filtering for $2 \mathrm{D}$ continuous systems with finite frequency specifications". International Journal of Systems Science, vol. 49, no. 1, pp. 43-57, 2017.

[13] Z.Duan, J. Zhou, and J. Shen. "Filter design for discrete-time two-dimensional T-S fuzzy systems with finite frequency specification". International Journal of Systems Science, vol. 50 no. 3, pp. 599-613, 2019.

[14] El-Amrani A., El Hajjaji A., Boukili B., Hmamed A., $" H_{\infty}$ Model Reduction for Two-Dimensional Discrete Systems in Finite Frequency Ranges", 26th Mediterranean Conference on Control and Automation (MED) (pp. 1-9). IEEE. 2018.

[15] D. Peaucelle. "Unified Formulation for Robust Analysis and Synthesis with Parameter Dependent Lyapunov Functions". doctoral thesis of Toulouse III University, France, 2000.

[16] YALMIP L. J., "A toolbox for modeling and optimization in MATLAB". Proceedings of the IEEE Computer-Aided Control System Design Conference, Taipei, Taiwan, Pp. 284-289, 2004.

[17] J. F. Sturm Using SeDuMi, "A MATLAB toolbox for optimization over symmetric cones". Optimization Methods and Software, pp. 625-653, 1999.

[18] Li, L., Wang, W., and Li, X. "New approach to $H_{\infty}$ filtering of two-dimensional T-S fuzzy systems". International Journal of Robust and Nonlinear Control, vol. 23, no. 17, pp. 1990-2012, 2013. 